\title{
Pink, Scaly, Annular Plaques in Concentric Rings Localized to Vitiliginous Patches
}

\author{
Jose A. Cervantes, MD; Kavina Patel, MD; Megan E. Shelton, MD; John F. Kaiser, MD
}

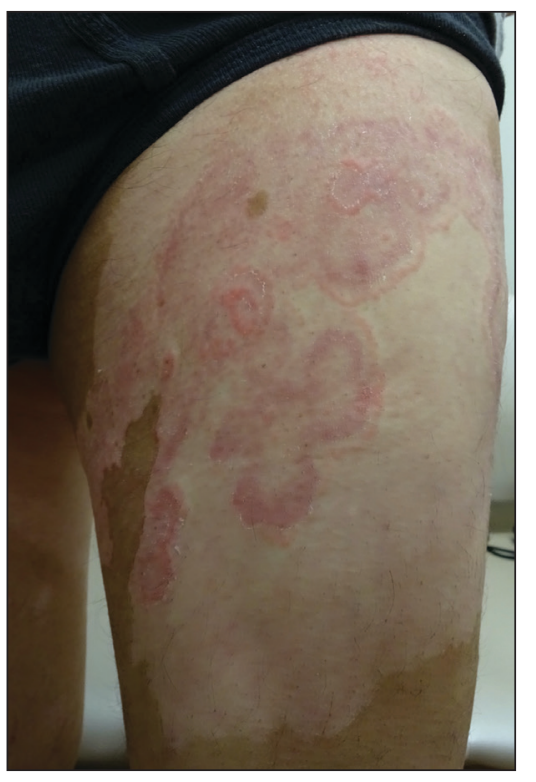

A 64-year-old man presented with generalized vitiligo. In addition to extensive depigmented macules, physical examination revealed the presence of onychomycosis and tinea corporis confirmed by microscopic examination of potassium hydroxide-treated superficial skin scrapings. Vitiligo treatment was postponed, and a 3-month course of oral terbinafine and naftifine cream was undertaken for the dermatophyte infections. Subsequent examination revealed that the patient's tinea corporis had improved, though there were localized areas of persistence. Given the patient's eagerness to treat his vitiligo, narrowband UVB phototherapy was started along with tolnaftate cream 1\% for treatment of the residual tinea corporis. After 2 months of narrowband UVB, partial repigmentation of the vitiligo was observed; however, he had developed extensive pink, scaly, annular plaques in concentric rings within residual vitiliginous patches on the lower extremities (top). Repeat examination of potassium hydroxide-treated skin scrapings revealed numerous hyphae (bottom). A fungal culture identified Trichophyton rubrum.

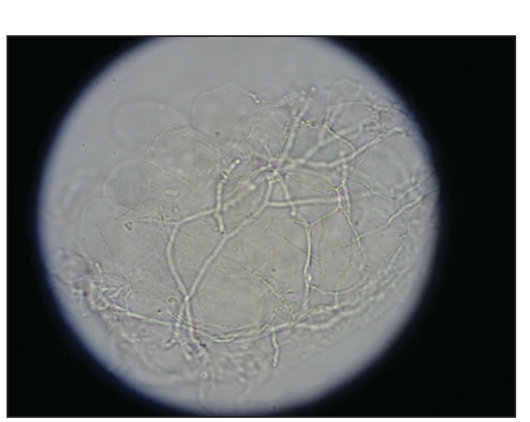

\section{WHAT'S YOUR DIAGNOSIS?}
a. erythema annulare centrifugum
b. erythema marginatum
c. erythema migrans
d. tinea imbricata
e. tinea pseudoimbricata

PLEASE TURN TO PAGE E29 FOR THE DIAGNOSIS

\footnotetext{
Drs. Cervantes and Patel are from Baylor College of Medicine, Houston, Texas. Dr. Patel is from the Department of Medicine. Dr. Shelton is from the Department of Dermatology, University of Michigan, Ann Arbor. Dr. Kaiser is from the Dermatology Section, Department of Veterans Affairs, Central Texas Veterans Health Care System, Austin Outpatient Clinic.

The authors report no conflict of interest.

The opinions expressed herein are those of the authors and do not necessarily reflect those of the US Government or any of its agencies. Correspondence: Jose A. Cervantes, MD (Josecervantes@email.Arizona.edu). doi:10.12788/cutis.0325
} 


\section{THE DIAGNOSIS:}

\section{Tinea Pseudoimbricata}

T inea pseudoimbricata and tinea indecisiva are synonyms describing cases of tinea corporis that manifest in scaly plaques in concentric rings evocative of those present in tinea imbricata. However, in contrast to tinea imbricata, cases of tinea pseudoimbricata are caused by dermatophytes other than Trichophyton concentricum. ${ }^{1}$ Tinea pseudoimbricata usually presents in association with immunosuppression, either systemic or local, and can be produced by application of topical medications such as corticosteroids. ${ }^{2}$ Mask-Bull et $\mathrm{al}^{3}$ reported the case of a 21-year-old man in the United States with no history of immunosuppressive conditions who presented with scaly erythematous annular plaques on the lateral neck that resolved with 2 pulsed doses of terbinafine. Potassium hydroxide preparation and fungal culture were both consistent with Trichophyton tonsurans. ${ }^{3}$

Trichophyton concentricum is an anthropophilic species of dermatophyte endemic to areas within the South Pacific, Southeast Asia, and Central and South America. Infection with $T$ concentricum produces tinea imbricata, which presents with concentric, scaly, annular rings. Cutaneous lesions of tinea imbricata caused by $T$ concentricum have a more generalized distribution and more densely grouped, concentric circles than the cutaneous findings seen in patients with tinea pseudoimbricata. ${ }^{4}$ Affected patients typically demonstrate negative delayed-type hypersensitivity to $T$ concentricum cytoplasmic antigen and T-lymphocyte hyporeactivity, which may contribute to the development of sequential waves of scaling observed in tinea imbricata. ${ }^{5}$

Trichophyton rubrum, the most common cause of tinea corporis, has been reported to cause some cases of tinea pseudoimbricata (indecisiva). ${ }^{1,2}$ It utilizes keratinases such as subtilisins (Sub3 and Sub4), leucine aminopeptidases (Lap1 and Lap2), and dipeptidyl peptidases (DppIV and $\mathrm{DppV}$ ) to invade the skin. Once inside, mannans, glycoprotein constituents of the cell wall, are released and bind to the cell surface of mononuclear phagocytes, subsequently moving into the cell by phagocytosis, thereafter interfering with RNA synthesis that is necessary for presentation of antigens to appropriate $\mathrm{T}$ cells and allowing for initiation of chronic infection. ${ }^{6,7}$ The cytotoxic response to superficial dermatophyte infection is triggered by major histocompatibility complex class I molecule activation of $\mathrm{CD} 8^{+}$cells. ${ }^{6,8}$

Our case is of interest given the localization of the superficial dermatophyte infection to only vitiliginous skin. This distribution and appearance while undergoing narrowband UVB (NB-UVB) treatment is rare. We postulate that our patient likely represents a case of locus minoris resistentiae, a phenomenon in which an area of skin exhibits a compromised immune microenvironment that predisposes it to disease. ${ }^{9}$
In vitiligo, NB-UVB modulates the immune response by increasing $\mathrm{IL}-10$, thereby promoting regulatory $\mathrm{T}$-cell differentiation with suppression of autoreactive $\mathrm{T}$ cells and induction of direct T-lymphocyte apoptosis. ${ }^{10,11}$ Although the mechanism accounting for our patient's presentation is unknown, we suspect NB-UVB-induced immunosuppression enabled persistence of the dermatophyte infection. The localization of the infection to the vitiliginous patches may result from the greater penetration of the UV light relative to the surrounding, normally pigmented skin. This relative difference in UV penetration would be expected to result in increased immunosuppression in the vitiliginous lesions and enhanced susceptibility to the fungal organisms.

Erythema annulare centrifugum is characterized by annular lesions with a trailing scale instead of the concentric rings seen in tinea pseudoimbricata. Erythema marginatum is seen in acute rheumatic fever and presents with a transient nonpruritic rash, usually on the trunk or extremities. Erythema migrans presents with fewer lesions that are less circinate in shape, and the patient often has a history of a tick bite. Tinea imbricata is caused by $T$ concentricum, while tinea pseudoimbricata is caused by $T$ tonsurans and other dermatophytes.

With the increasing use of immunosuppressant drugs, the prevalence of tinea pseudoimbricata is hypothesized to increase. ${ }^{1}$ The presence of tinea pseudoimbricata should alert dermatologists to the possible overuse of topical corticosteroids, and other forms of immunosuppression also should be considered.

\section{REFERENCES}

1. Lim SP, Smith AG. "Tinea pseudoimbricata": tinea corporis in a renal transplant recipient mimicking the concentric rings of tinea imbricata. Clin Exp Dermatol. 2003;28:332-333.

2. Batta K, Ramlogan D, Smith AG, et al. 'Tinea indecisiva' may mimic the concentric rings of tinea imbricata. Br J Dermatol. 2002;147:384.

3. Mask-Bull L, Patel R, Tarbox MB. America's first case of tinea pseudoimbricata. Am J Dermatol Venereol. 2015;4:15-17.

4. Meena M, Mittal A. Tinea pseudo-imbricata. J Assoc Physicians India. 2018;66:79

5. Hay RJ, Reid S, Talwat E, et al. Immune responses of patients with tinea imbricata. Br J Dermatol. 1983;108:581-586.

6. Dahl MV. Suppression of immunity and inflammation by products produced by dermatophytes. J Am Acad Dermatol. 1993;28(5 pt 1):S19-S23.

7. Blutfield MS, Lohre JM, Pawich DA, et al. The immunologic response to Trichophyton rubrum in lower extremity fungal infections. J Fungi (Basel). 2015;1:130-137.

8. De Hoog S, Monod M, Dawson T, et al. Skin fungi from colonization to infection [published online July 2017]. Microbiol Spectr. doi:10.1128/ microbiolspec.FUNK-0049-2016

9. Lo Schiavo A, Ruocco E, Russo T, et al. Locus minoris resistentiae: an old but still valid way of thinking in medicine. Clin Dermatol. 2014;32:553-556

10. Ponsonby AL, Lucas RM, van der Mei IA. UVR, vitamin D and three autoimmune diseases-multiple sclerosis, type 1 diabetes, rheumatoid arthritis. Photochem Photobiol. 2005;81:1267-1275. 
11. Yazdani Abyaneh M, Griffith RD, Falto-Aizpurua L, et al. Narrowband ultraviolet B phototherapy in combination with other therapies for vitiligo: mechanisms and efficacies. J Eur Acad Dermatol Venereol. 2014;28:1610-1622 